\title{
Dark Energy and the Accelerated Expansion of the Universe
}

\author{
Ioav Waga \\ Universidade Federal do Rio de Janeiro, Instituto de Física \\ Rio de Janeiro, RJ, 21945-970, Brazil \\ E-mail: ioav@if.ufrj.br \\ Received 7 January, 2000
}

\begin{abstract}
Recent observations indicate that the expansion of the Universe is accelerating. This suggests the existence of some kind of exotic matter with negative pressure. The simplest possibility is a cosmological constant but there are alternatives, as for instance an evolving scalar field. In this paper we explore constraints from lensing statistics and high-z type Ia Supernovae on some of these alternatives.
\end{abstract}

\section{Introduction}

"I am a detective in search of a criminal - the cosmical constant. I know he exists, but I do not know his appearance; for instance $I$ do not know if he is a little man or a tall man. Naturally the first move of my chief (de Sitter) was to order a search for footprints, or what look like footprints...

... I think I have now about enough evidence to justify an arrest."

Arthur Eddington in "The Expanding Universe".

Recent observations of type Ia supernovae (SneIa) suggest that the expansion of the Universe is accelerating $[1,3]$. In fact what it is observed is that high-z SneIa - that after corrections are almost perfect standard candles - are fainter than would be expected in a Universe where the expansion is slowing down or remain constant. Although there are still some possible sources of systematic effects (source evolution and dust are those that concern most), the current accepted explanation is that they appear fainter because in a Universe that is speeding up distances are larger. To get a qualitative understanding of why accelerated expansion leads to larger distances consider a nearby source with measured redshift $z$. Forget for a while peculiar velocities and think on redshift as a simple Doppler effect. In this approximation, for nearby sources the measured redshift can be thought as giving us the velocity of the photon source at the time it was emitted $\left(z=v_{e} / c\right)$. Now consider the velocity-distance law [4]: $v(t)=H(t) d(t)$, where $v$ is the value of the source velocity and $d$ its proper distance at the same time $t$. Let us consider $t$ the present time. So, if in the past the photon source had Hubble flow velocity $v_{e}$ and the rate of the Uni- verse expansion is speeding up, the present value of the source velocity is larger now than it would be in case the Universe expands with, for instance, constant velocity. So, assuming the same value for the Hubble parameter today, it follows from the velocity-distance law, that a larger velocity implies a larger distance. It follows from Friedman equation $(\ddot{a} / a=-(4 \pi G / 3)(\rho+3 p))$ that accelerated expansion may be achieved if the Universe has a dominant component with an effective negative pressure. Dark energy, dynamical- $\Lambda$ (dynamical vacuum energy) or quintessence are different names that have been used to denote this component. A cosmological constant is its simplest form.

Recent studies incorporating new CMB data $[5,6]$ confirm previous analysis suggesting a large value for the total density parameter $\left(\Omega_{\text {total }}>0.4\right)$ and favor a nearly flat Universe. Further, a different set of observations [7] now unambiguously indicate low values $\left(\Omega_{m_{0}}=0.3 \pm 0.1\right)$ for the matter density parameter $\left(\Omega_{m 0}\right)$. In combination these two results are in line with the conventional interpretation of the SneIa results and all together strongly support flat cosmologies with $\Omega_{m 0} \sim 0.3$ and a dark energy component with $\Omega_{X} \sim 0.7$. These models are also theoretically appealing since a smooth component on small scales (20-30 $\left.h^{-1} \mathrm{Mpc}\right)$ reconcile inflation with the low values for $\Omega_{m 0}[8]$.

Since the beginnings of modern cosmology, the cosmological constant $(\Lambda)$ re-appears from time to time. Historically, Einstein [9] himself was the first one to include a cosmological constant in the general relativity field equations in order to make them compatible with a static universe. Lemaitre [10] also made use of $\Lambda$ to 
show how the age of the Universe could be greater than the inverse value of the Hubble parameter accepted at that time. The cosmological term was also kept different from zero by Eddington [11], who preferred the cosmological expansion starting from an Einstein static universe. Petrosian, Salpeter and Szekeres [12] introduced this term to account for an apparently observed preponderance of sources with redshift $z \simeq 2$, and Gunn and Tinsley [13] invoked it in 1975 to explain a seemingly accelerated expansion of the universe. For a review on the early history of the cosmological constant see North [14]. Several aspects of non null $\Lambda$ cosmologies such as the age problem, classification of models, classical tests, observational constraints on $\Lambda$, structure formation and gravitational lenses were discussed by several authors. For a review and/or an extensive list of references see [20].

In the past the cosmological constant has been introduced several times to reconciliate theory with observations. When better data became available or improved interpretation showed it was not needed, $\Lambda$ has always been discarded. However, now it is possible that this situation will change and that the "genie" will remain, perhaps for ever, out of the bottle [19]. According to quantum field theory, the vacuum state has zero-point fluctuations to whose energy the gravitational field is sensitive. As is well known, Lorentz invariance implies that the vacuum contribution to the energy-momentum tensor must be of the form $\rho_{\Lambda} g_{\mu \nu}$, where $g_{\mu \nu}$ is the metric tensor. Therefore, quantum field theory predicts a cosmological term that added to the bare cosmological constant gives rise to an effective cosmological term. The problem then lies in the maximum value that observations indicate to this term,

$$
\rho_{\Lambda} \lesssim \frac{3 m_{p l}^{2} H_{0}^{2}}{8 \pi},
$$

or

$$
\Lambda \lesssim 10^{-56} \mathrm{~cm}^{-2}
$$

The above upper limit is 50 to 120 orders of magnitude below the estimate given by quantum field theory. The difficulty in explaining the smallness of the effective $\Lambda$ is known as the "cosmological constant problem". One of the original motivations for introducing the idea of a dynamical $\Lambda$-term was to alleviate this problem. There are also observational motivations. For instance, in these models the COBE normalized amplitude of the mass power spectrum is in general lower than in the conventional constant- $\Lambda$ model, in accordance with observations [24]. Further, the distance to an object with redshift $z$ is smaller than the distance to the same object in a constant- $\Lambda$ model (assuming the same value of $\Omega_{m 0}$ ). So, constraints coming from lensing statistics are weaker in these models [15, 18].

The dynamical- $\Lambda$ models present in the literature can schematically be divided in three types: scalar field $[31,23,24,26,25]$, x-fluid $[29,30,18]$ and decaying- $\Lambda$ laws $[28,17,16]$. A phenomenological decaying- $\Lambda$ law model in which $\Lambda$ decreases as $\Lambda \propto a^{-m}$ [here $a$ is the scale factor of the Friedman-Robertson-Walker (FRW) metric and $m$ is a constant $(0 \leq m<3)]$ was suggested in Refs. $[17,16]$. It was observed that the Einstein equations for these models are the same if instead of a $\Lambda^{-}$ term, it would be considered (beside matter and radiation) a x-fluid with equation of state, $p_{x}=\left(\frac{m}{3}-1\right) \rho_{x}$. In spite of the similarity at the level of Einstein equations, these two phenomenological models are different. For instance, in the case of a decaying $\Lambda$-term, matter is created as a result of the decaying vacuum, while in the exotic fluid description the $\mathrm{x}$-component is conserved.

In this paper we shall deal with two cosmological tests: gravitational lensing statistics and the SneIa magnitude redshift test. As mentioned before the strongest observational support for an accelerated universe comes from SneIa. This test can be considered the main motivation for introducing some kind of exotic matter with negative pressure. On the other hand, most lensing statistics analysis give lower values for $\Lambda$ and we find interesting to compare the predictions of these two important tests. Here we first consider the special case where the exotic component is a $\mathrm{x}$-fluid with constant equation of state and that is smooth on scales smaller than horizon. We also report constraints from gravitational lensing statistics and high-z SneIa on two representative scalar field potentials that give rise to effective decaying $\Lambda$ models: PNGB potentials $\left(V(\phi)=M^{4}(1+\cos (\phi / f))\right)$ and inverse power-law potentials $\left(V(\phi)=M^{4+\alpha} \phi^{-\alpha}\right)$. There are different motivations for introducing these two potentials. The best motivation for the PNGB potential comes from particle physics while the inverse power-law potentials have the property of "tracking" that allow the field to start with a wide set of initial conditions [32].

Let us consider first the motivations for introducing the PNGB potential[21, 23, 25]. In order to act approximately like a cosmological constant at recent epochs with $\Omega_{\phi} \sim 1$, the potential energy density should be of order the critical density, $M^{4} \sim 3 H_{0}^{2} m_{P l}^{2} / 8 \pi$, or $M \simeq 3 \times 10^{-3} h^{1 / 2} \mathrm{eV}$. As ususal we set $V=0$ at the minimum of the potential by the assumption that the fundamental vacuum energy of the Universe is zero, for reasons not yet understood. Further, since observations indicate an accelerated expansion, at present time the field kinetic energy must be relatively small compared to its potential energy. This implies that the 
motion of the field is still (nearly) overdamped, that is, $\sqrt{\left|V^{\prime \prime}\left(\phi_{0}\right)\right|} \lesssim 3 H_{0}=5 \times 10^{-33} h \mathrm{eV}$. The two conditions above imply that $f \sim m_{P l} \simeq 10^{19} \mathrm{GeV}$. Further, the PNGB mass is $m_{\phi} \sim M^{2} / f \simeq 10^{-32} h \mathrm{eV}$. In quantum field theory, such ultra-low-mass scalars are not generically natural: radiative corrections generate large mass renormalizations at each order of perturbation theory. To incorporate ultra-light scalars into particle physics, their small masses should be at least 'technically' natural, that is, protected by symmetries, such that when the small masses are set to zero, they cannot be generated in any order of perturbation theory, owing to the restrictive symmetry.

From the viewpoint of quantum field theory, pseudoNambu-Goldstone bosons (PNGBs) are the simplest way to have naturally ultra-low mass, spin-0 particles. PNGB models are characterized by two mass scales, a spontaneous symmetry breaking scale $f$ (at which the effective Lagrangian still retains the symmetry) and an explicit breaking scale $M$ (at which the effective Lagrangian contains the explicit symmetry breaking term). Thus, the two dynamical conditions on $f$ and $M$ above essentially fix these two mass scales. Note that $M \sim 10^{-3} \mathrm{eV}$ is close to the neutrino mass scale for the MSW solution to the solar neutrino problem, and $f \sim m_{P l} \simeq 10^{19} \mathrm{GeV}$, the Planck scale. Since these scales have a plausible origin in particle physics models, we may have an explanation for the 'coincidence' that the vacuum energy is dynamically important at the present epoch [23, 21, 22]. Moreover, the small mass $m_{\phi}$ is technically natural.

Next consider the inverse power-law case. The best motivation for introducing this potential is the existence of attractor (tracking) solutions such that if $\rho_{\phi} \ll \rho_{B}$, the following relationship is satisfied: $\rho_{\phi}^{T R} \sim a^{3\left(\gamma_{B}-\gamma_{\phi}^{T R}\right)} \rho_{B}$ with $\gamma_{\phi}^{T R}=\gamma_{B} \alpha /(2+\alpha)<\gamma_{B}$ $[27,15,32]$. Here $a$ is the scale factor of the FRW metric and $\gamma_{B}$ stands for the adiabatic index of the background $\left(\gamma_{B}=4 / 3\right.$ during the radiation dominated era and $\gamma_{B}=1$ during the matter dominated era (MDE)). So, if the field is on track its energy density decreases slower than the background energy density and the field eventually will begin to dominate the dynamics of the expansion. If the field is on track during the MDE, its effective adiabatic index will be less than unity and the field effective pressure will be negative. This condition by itself does not guaranty accelerated expansion. It is necessary that the field dominates the dynamics and that the total effective adiabatic index be smaller than 2/3. However, for inverse power-law potentials at late times $\Omega_{\phi} \rightarrow 1$, such that when the growing
$\Omega_{\phi}$ starts to become non negligible, $\gamma_{\phi}$ deviates from the above tracking value decreasing toward the value $\gamma_{\phi} \rightarrow 0$. So, even if $\alpha>4$ such that initially $\gamma_{\phi}^{T R}>2 / 3$ in the MDE, as the field dominates and $\gamma_{\phi}$ decreases, the Universe will enter in a phase of accelerated expansion. If $\Omega_{m 0}$ and $\alpha$ are sufficiently low this will happen before the present time. For inverse power-law potentials the two conditions $\Omega_{\phi 0} \sim 1$ and the preponderance of the field potential energy over its kinetic energy $\left(\sqrt{\left|V^{\prime \prime}\left(\phi_{0}\right)\right|} \lesssim 3 H_{0}\right)$ imply $M \sim 10^{\frac{27 \alpha-12}{\alpha+4}} \mathrm{eV}$ and $\phi_{0} \sim m_{P l}$. Since $\phi_{0} \sim m_{P l}$ quantum gravitational corrections corrections to the potential are important and could invalidate this picture [33].

In the very early Universe, in order to successfully achieve tracking the field energy density must be smaller than the radiation energy density. If in addition it is smaller than the initial value of the tracking energy density it will remain frozen until they have comparable magnitude and then the field starts to follow the tracking solution. Otherwise, if it is larger than the initial value of the tracking energy density, it will enter in a phase of kinetic energy domination $\left(\gamma_{\phi} \sim 2\right)$, $\rho_{\phi}$ decreases fast $\left(\rho_{\phi} \propto a^{-6}\right)$ overshooting the tracker solution. After that, as in the previous case, the field frozen and again when the tracking energy density and $\rho_{\phi}$ have comparable magnitude the field begins to follow the tracking solution. Whatever is the case there is always a phase before tracking in which the field is frozen. So, an important point is the value of the field energy density when it freezes. For instance, is it smaller or larger than $\rho_{e q}$, the energy density at radiation and nonrelativistic matter equality? Did the field had time to completely achieve tracking or not? In fact the constraints imposed by cosmological tests on the parameter space depend on this condition.

We report constraints from lensing statistics and high-z Sne for the inverse power-law potential starting from two different set of initial conditions. In the first one we assume that the field is frozen by the matterradiation equality epoch. This is the approach we followed in Ref. [25]. In this case depending on the value of $\alpha$ and $\Omega_{m 0}$, it may happen that the field does not have time to reach the tracking solution. In general, if $\Omega_{m 0}$ is large we observe that $\gamma_{\phi}$ is still growing by the present time, away from its initial value $\gamma_{\phi}=0$. Otherwise, if $\Omega_{m 0}$ is sufficiently low, $\gamma_{\phi}$ will reach a maximum value (not necessarily its tracking value) in the past and, by the present time, will be decreasing to the final attractor value $\gamma_{\phi}=0$. In our second approach we assume that the field starts tracking very early in the Universe evolution. ${ }^{1}$ When $\rho_{\phi}$ becomes

\footnotetext{
${ }^{1}$ In fact this is true only if $\alpha$ is not very close to zero. The case $\alpha=0$ is equivalent to a cosmological constant and the field remains
} 
non-negligible, $\gamma_{\phi}$ starts to decrease to its final attractor value $\gamma_{\phi}=0$. Recently constraints from high-z Sne on power-law potentials with the field rolling with this set of initial conditions were obtained by Podariu and Ratra[34]. We complement their analysis including the lensing constraints as well.

This paper is based on Refs.[23, 25, 18, 46] and is organized as follows. In Sec.II we present our statistical lensing approach. The methods used to obtain constraints from SneIa observations is briefly described in Sec.III. In Sec.IV our main results are presented and in Sec. $V$ conclusions are stressed out.

\section{Lensing statistics}

In this section we outline our statistical lensing approach. It is based on Refs:[39, 40] and is described with some more detail in [18]. We start defining the following likelihood function, [39]

$$
\mathcal{L}_{\text {lens }}=\prod_{i=1}^{N_{U}}\left(1-p_{i}^{\prime}\right) \prod_{j=1}^{N_{L}} p_{j}^{\prime} \prod_{k=1}^{N_{L}} p_{c k}^{\prime}
$$

Here $N_{L}$ is the number of quasars that have multiple image, $N_{U}$ is the number of quasars that don't have, $p_{i}^{\prime} \ll 1$ is the probability that quasar $i$ is lensed and $p_{c k}^{\prime}$ is the configuration probability, that we shall consider as the probability that quasar $k$ is lensed with the observed image separation. To perform the statistical analysis we use data from the HST Snapshot survey (498 high luminous quasars (HLQ), the Crampton survey (43 HLQ), the Yee survey ( $37 \mathrm{HLQ}$ ), the ESO/Liege survey (61 HLQ), The HST GO observations (17 HLQ), the CFA survey (102 HLQ), and the NOT survey (104 HLQ) [35]. We considered a total of $862(z>1)$ high luminous optical quasars plus 5 lenses.

The differential probability, $d \tau$, that a line of sight intersects a galaxy at redshift $z_{L}$ in the interval $d z_{L}$ from a population with number density $n_{G}$ is,

$$
d \tau=c n_{G} \pi a_{e r}^{2} d t
$$

where $a_{c r}$ is the maximum distance of the lens from the optical axes for which multiple images are possible. It is a function of the angular diameter distance between observer and lens, lens and source, observer and source and it also depends on the lens model.

In our approach we use a singular isothermal sphere (SIS) as the lens model, we neglect lensing by spiral galaxies, assume conserved comoving number density of early tipe galaxies, $n_{e}=n_{0}(1+z)^{3}$ and a Schechter form for the galaxy population,

$$
n_{0}=\int_{0}^{\infty} n_{*}\left(\frac{L}{L^{*}}\right)^{\alpha} \exp \left(-\frac{L}{L^{*}}\right) \frac{d L}{L^{*}},
$$

with $n_{*}=0.61 h^{3} 10^{-2} \mathrm{Mpc}^{-3}$ and $\alpha=-1.0$. We assume that the luminosity satisfies the Faber-Jackson relation [37], $L / L^{*}=\left(\sigma_{\|} / \sigma_{\|}^{*}\right)^{\gamma}$, with $\gamma=4$ and take $\sigma_{\|}^{*}=225 \mathrm{Km} / \mathrm{s}$.

The total optical depth $(\tau)$, obtained by integrating $d \tau$ along the line of sight from 0 to $z_{S}$, can be expressed analytically,

$$
\tau\left(z_{S}\right)=\frac{F}{30}\left(d_{A}\left(0, z_{S}\right)\left(1+z_{S}\right)\right)^{3}\left(c H_{0}^{-1}\right)^{-3}
$$

where $F=16 \pi^{3} n_{e}\left(c H_{0}^{-1}\right)^{3}\left(\sigma_{\|}^{*} / c\right)^{4} \Gamma(1+\alpha+4 / \gamma) \simeq$ 0.026 measures the effectiveness of the lens in producing multiple images [36].

It is important to include two corrections to the optical depth: magnification bias and selection function due to finite resolution and dynamic range [39].

Since lensing increase the apparent brightness of a quasar and since there are more faint quasars than bright ones, there will be over representation of lensed quasars in a flux limited sample. The bias factor is given by $[38,39,40]$

$$
\mathbf{B}(m, z)=M_{0}^{2} \mathcal{B}\left(m, z, M_{0}, M_{2}\right),
$$

where

$$
\begin{aligned}
& \mathcal{B}\left(m, z, M_{1}, M_{2}\right)= \\
& 2\left(\frac{d N_{q}}{d m}\right)^{-1} \int_{M_{1}}^{M_{2}} \frac{d M}{M^{3}} \frac{d N_{q}}{d m}(m+2.5 \log M, z) .
\end{aligned}
$$

Since we are modeling the lens by a SIS profile, $M_{0}=2$, and we use $M_{2}=10^{4}$ in the numerical computation.

We use the following expression for the quasar luminosity function [40]

$$
\frac{d N_{q}}{d m} \propto\left(10^{-a(m-\bar{m})}+10^{-b(m-\bar{m})}\right)^{-1},
$$

where

$$
\bar{m}=\left\{\begin{array}{lrr}
m_{0}+(z+1) & \text { for } & z<1 \\
m_{0} & \text { for } & 1<z<3 \\
m_{0}-0.7(z-3) & \text { for } & z>3
\end{array}\right.
$$

and we assume $a=1.07, b=0.27$ and $m_{0}=18.92$. The magnification corrected probabilities are

$$
p_{i}=\tau\left(z_{i}\right) \mathbf{B}\left(m_{i}, z_{i}\right)
$$

Finally we have to consider the selection function due to finite resolution and dynamic range. It can be 
shown that the selection function corrected probabilities are: [39]

$$
p_{i}^{\prime}(m, z)=p_{i} \frac{\int d \theta p_{c}(\theta) \mathcal{B}\left(m, z, M_{f}(\theta), M_{2}\right)}{\mathcal{B}\left(m, z, M_{0}, M_{2}\right)},
$$

and

$$
p_{c i}^{\prime}=p_{c i}(\theta)\left(\frac{p_{i}}{p_{i}^{\prime}}\right) \frac{\mathcal{B}\left(m, z, M_{f}(\theta), M_{2}\right)}{\mathcal{B}\left(m, z, M_{0}, M_{2}\right)},
$$

where

$$
\begin{aligned}
& p_{c}(\theta)=\frac{F}{\tau\left(z_{S}\right)} \int_{0}^{z_{S}}\left(1+z_{L}\right)^{3} \\
& \times\left(\frac{d_{A}\left(0, z_{L}\right) d_{A}\left(z_{L}, z_{S}\right)}{c H_{0}^{-1} d_{A}\left(0, z_{S}\right)}\right)^{2} 8 \pi\left(\frac{\sigma_{\|}^{\star}}{c}\right)^{2} \\
& \times\left(-\frac{1}{c H_{0}^{-1}} \frac{c d t}{d z_{L}}\right) \frac{\gamma / 2}{\Gamma\left(\alpha+1+\frac{4}{\gamma}\right)} \\
& \times\left(\frac{d_{A}\left(0, z_{S}\right)}{d_{A}\left(z_{L}, z_{S}\right)} \frac{\theta}{8 \pi\left(\frac{\sigma_{\Perp}^{\star}}{c}\right)^{2}}\right)^{\frac{\gamma}{2}\left(\alpha+1+\frac{4}{\gamma}\right)} \\
& \times \exp \left[-\left(\frac{d_{A}\left(0, z_{S}\right)}{d_{A}\left(z_{L}, z_{S}\right)} \frac{\theta}{8 \pi\left(\frac{\sigma_{\| I}^{\star}}{c}\right)^{2}}\right)^{\frac{\gamma}{2}}\right] \frac{1}{\theta} d z_{L}, \\
& M_{f}(\theta)=M_{0} \frac{1+f}{f-1}, \quad f>1,
\end{aligned}
$$

and

$$
f=f(\theta)=10^{0.4 \Delta m(\theta)}
$$

To simplify computation we use two selection functions [39], one for the HST observations and another one for all the ground based surveys. Using more accurate selection functions for each ground based observations separately have little statistical effect.

Recently Falco et al. [41] observed that statistical lensing analysis based on optical and radio observations can be reconciled if the existence of dust in $\mathrm{E} / \mathrm{SO}$ galaxies is considered. In our computation we assume a mean extinction of $\Delta m=0.5 \mathrm{mag}$ as suggested by their estimates. Current statistical analysis using both HLQ and radio sources tight the constraints on a cosmological constant. Although this combined analysis for dynamical- $\Lambda$ models is still in progress we can have an idea of what should be expected if, for instance, we reduce extinction in our analysis to $\Delta m=0.3 \mathrm{mag}$. In this case the new $2 \sigma$ contours, shift by approximately $1 \sigma$ from the previous one, that is, the new $2 \sigma$ contours will be located slightly before the $1 \sigma$ contours of the figures in Sec.4.
By expressing $\mathcal{L}_{\text {lens }}$ as a function of the parameters $m$ and $\Omega_{m 0}$ we obtained the maximum of the likelihood function $\left(\mathcal{L}_{\text {lens }}^{\text {max }}\right)$ and formed the ratio $l=\mathcal{L}_{\text {lens }} / \mathcal{L}_{\text {lens }}^{\text {max }}$. It can be shown that with two parameters, the distribution of $-2 \ln l$ tends to a $\chi^{2}$ distribution with two degrees of freedom [39].

\section{High-redshift type Ia Super- novae [18]}

There are two major ongoing programs to systematically search and study high-z supernovae. Although the very preliminary results indicated a low value for the cosmological constant ( $\Omega_{\Lambda}<0.51$ at the $95 \%$ confidence level) [43], more recent analysis with larger sample of supernovae, now points to a different direction. Now the data indicate an accelerated expansion such that $\Omega_{\Lambda} \sim 0.7, \Omega_{m 0} \sim 0.3$ and strongly supports a flat Universe.[1, 2].

In our analysis we consider data from the High-z Supernovae Search Team. We use the 27 low- $z$ and 10 high-z SneIa (we include SN97ck) reported in Riess et al. [1] and consider data with the MLCS [44, 1] method applied to the supernovae light curves. Following a procedure similar to that described in Riess et al.[1], for each model,we determine the cosmological parameters $\hat{\theta}_{a}$ through a $\chi^{2}$ minimization neglecting the unphysical region $\Omega_{m 0}<0$. To simplify computation we fix the Hubble parameter to $H_{0}=65.2 \mathrm{~km} / \mathrm{s} \mathrm{Mpc}^{-1}$ [1], but the results are independent of this choice for $H_{0}$ [1]. We use

$$
\chi_{s n e}^{2}\left(\hat{\theta}_{a}\right)=\sum_{i=1}^{37} \frac{\left(\mu_{p}\left(z_{i}, \hat{\theta}_{a}\right)-\mu_{0, i}\right)^{2}}{\sigma_{\mu_{0, i}}^{2}+\sigma_{v i}^{2}}
$$

where

$$
\mu_{p}=5 \log d_{L}+25,
$$

is the distance modulus predicted by each model, $\mu_{0}$ is the observed (after corrections) distance modulus, $\sigma_{\mu_{0}}$ its uncertainty and $\sigma_{v}$ is the dispersion in galaxy redshift due to peculiar velocities. Following [1] we use $\sigma_{v i}=\frac{5}{\ln 10} \frac{200 \mathrm{~km} / \mathrm{s}}{c z_{i}}$ and for high-z SneIa with z not derived from emission lines in the host galaxy, we add $2500 \mathrm{~km} / \mathrm{s}$ in quadrature to $200 \mathrm{~km} / \mathrm{s}$ (see Table 1 in $[1])$.

\section{Results $[18,46]$}

"The genie has been let out of the bottle, and it is no longer easy to force it back in" [19] The cosmological constant and the theory of elementary particles 
Ya. B. Zeldovich

We first consider the $\mathrm{x}$-fluid model. In Fig.1 we present contours of constant likelihood $95.4 \%(2 \sigma)$ and $68 \%(1 \sigma)$ arising from the $\Delta \chi_{s n e}^{2}$ analysis together with those from lensing (dashed lines). For SneIa the peak of the likelihood is located at $m \simeq 1.1$ and $\Omega_{m 0}=0$. If we fix $m=0$ we get $\Omega_{m 0}=0.25 \pm 0.08(1 \sigma)$. For lensing the maximum of the likelihood occurs for $m \simeq 2.4$ and $\Omega_{m 0}=0$. The same approach when applied to constant $\Lambda$ models (since $m=0$ we now have only one degree of freedom) gives: $\Omega_{\Lambda} \lesssim 0.76$ (or $\Omega_{m 0} \gtrsim 0.24$ ) at $2 \sigma$, $1 \gtrsim \Omega_{m 0} \gtrsim 0.39$ at $1 \sigma$ with a best fit at $\Omega_{m 0} \simeq 0.61$. From the figure it is clear that there is a region in the parameter space (the region inside the triangle with vertices $\left(m \simeq 0.85, \Omega_{m 0} \simeq 0.24\right),\left(m=0, \Omega_{m 0} \simeq 0.32\right)$ and $\left(m=0, \Omega_{m 0} \simeq 0.38\right)$ ) such that all points are inside the $1 \sigma(68 \%)$ confidence region of both tests.

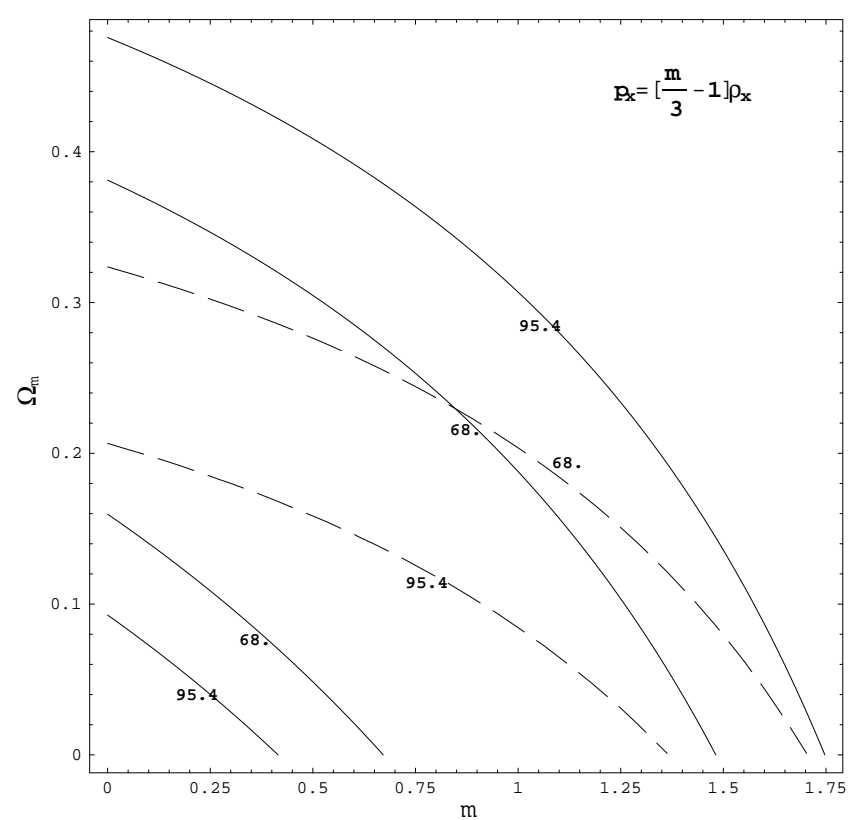

Figure 1. Contours of constant likelihood (95.4\% and $68 \%$ ) arising from lensing statistics (dashed lines) and type Ia supernovae are shown for the $\mathrm{x}$-fluid model.

In Fig. 2 we display contours (95.4\% and $68 \%$ ) of the combined (lensing plus SneIa) likelihood. For the combined $\chi^{2}$ analysis we used $\chi_{\text {tot }}^{2}=\Delta \chi_{s n e}^{2}-2 \ln l$, with $l=\mathcal{L}_{\text {lens }} / \mathcal{L}_{\text {lens }}^{\max }$ as defined in Sec.2. Although the peak of the likelihood for each test separately occurs at $\Omega_{m 0}=0$, the maximum of the combined likelihood occurs at $m=0$ (cosmological constant) and $\Omega_{m 0} \simeq 0.33$. Note that best fit models of the combined likelihood are in accelerated expansion $\left(q_{0}<0\right)$. Models with $m=2$ (cosmic strings [29]) and any value of $\Omega_{m 0}$ are at more than $99 \%$ c.l. away from the peak of the likelihood. We observe that if, for instance, we take $h=0.65$ and $\Omega_{B} h^{2}=0.02$, the CMBR first acoustic peak ( $\left.\ell_{\text {peak }}\right)$, models with $m$ and $\Omega_{m 0}$ inside the $1 \sigma$ allowed region in Fig 2 , will have $\ell_{\text {peak }}$ values between $\simeq 215$ and 230 , (see Fig. 4 in Ref.[30]), that are close to the current best values for $\ell_{\text {peak }}$ obtained from CMBR data. Models with parameters $m$ and $\Omega_{m 0}$ in this region are in agreement with the current CMBR data as well. Constraints from observations of clusters suggest $\Omega_{m 0}>0.15$ [7]. In Fig. 2 we display this constrain as a dotted line. Models above this line are preferred.

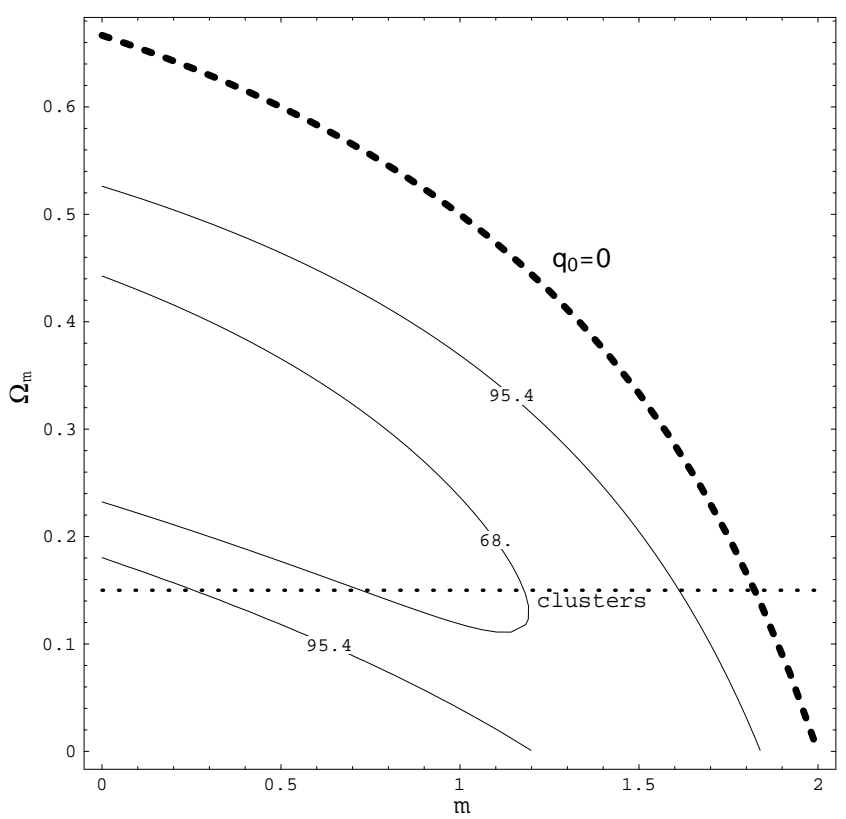

Figure 2. Contours of combined likelihood (95.4\% and $68 \%$ ) arising from lensing statistics and type Ia supernovae are shown for the $\mathrm{x}$-fluid model.

Now we consider the scalar field models. In Fig.3 we show the $95.4 \%$ and $68.3 \%$ C. L. limits from lensing (short dashed contours) and the SNe Ia data on the parameters $f$ and $M$ of the PNGB potential. As in [25], these limits apply to models with the initial condition $\frac{4 \sqrt{\pi} \phi\left(t_{i}\right)}{m_{P l}}=1.5$ and $\frac{d \phi}{d t}\left(t_{i}\right)=0$, with $t_{i}=10^{-5} t_{0}$; for other choices, the bounding contours would shift by small amounts in the $f-M$ plane. We also plot some contours of constant $\Omega_{m 0}$ (dashed) and the curve $q_{0}=0$ (long dashed contour) as a function of the parameters $f$ and $M$. The best fit region of the parameter space is limited by the lensing and SneIa $95.4 \%$ C. L. contours and also by $\Omega_{m 0}>0.15$, that we took as our lower limit for $\Omega_{m 0}$. The lensing $2 \sigma$ contour roughly coincides with the density parameter contour $\Omega_{m 0} \sim 0.2$. The data clearly favors accelerated expansion (the region above the $q_{0}=0$ curve) but curiously there is a small region in the parameter space, close to the protuberance of the best-fit area (that is allowed at the $2 \sigma$ C.L.), where the Universe is not in accelerated expansion at the present time. This area disappears if $\Omega_{m 0}>0.3$. The data 
also favor the region in the parameter space where the field is still nearly frozen, that is, the region between the almost vertical lines of the $2 \sigma$ lensing and SneIa contours.

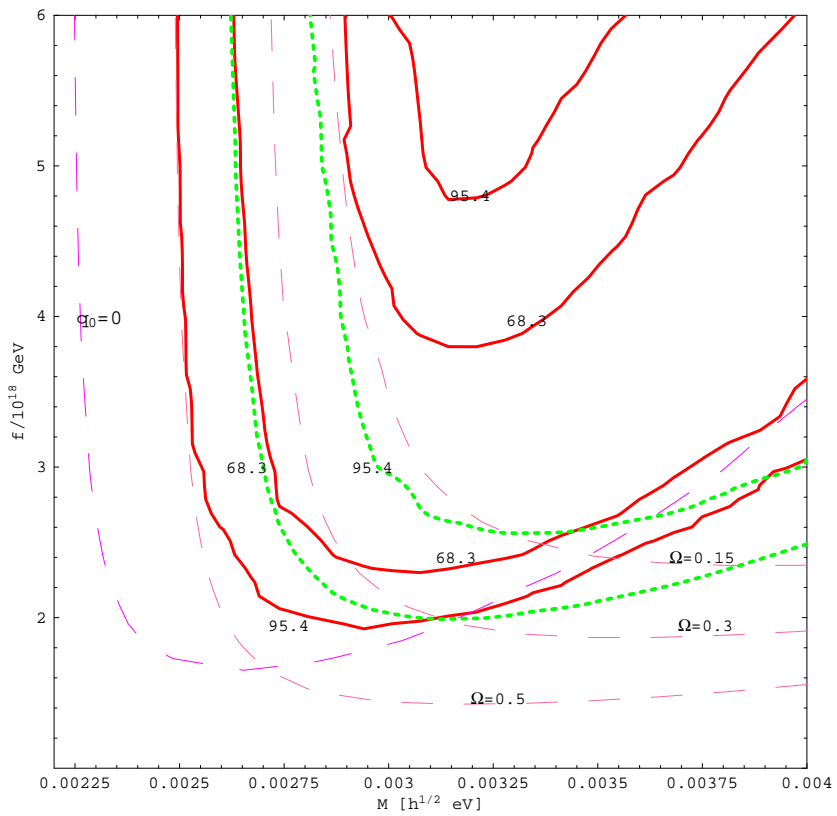

Figure 3. Contours of constant likelihood (95.4\% and $68 \%$ ) arising from lensing statistics (short dashed lines) and type Ia supernovae are shown for the PNGB model.

In Figs.4 and 5 we show the $95.4 \%$ and $68.3 \% \mathrm{C}$. L. limits from lensing (thick dashed contours) and the SNeIa data on the parameters $\alpha$ and $\Omega_{m 0}$ of the inverse power-law potential. For Fig. 4 we assume that the field is frozen around the matter-radiation equality epoch, while for Fig.5 the field starts tracking early in the Universe evolution. Again we display in the figures, as an horizontal straight line, $\Omega_{m 0}=0.15$ that we took as our lower limit for $\Omega_{m 0}$. We also plot some contours with the present value of the equation of state $w_{0}=\gamma_{0}-1$ (thin dashed contours) and the curve $q_{0}=0$ (long dashed contour). The present data exclude $\alpha>5$ for the two initial data set we used. We also have $w_{0} \lesssim-0.5$ that is also roughly what we got in the $\mathrm{x}$-fluid case, that sometimes is used as an approximation for the inverse power-law potential. We also observe that the lensing constraints on the equation of state are weak, constraining only low values of $\Omega_{m 0}$ and $\alpha$. Although weak they are consistent with the Snela constraints. We can tight the constraints on the equation of state if we consider a higher value for the $\Omega_{m 0}$ lower bound. For instance, if we take $\Omega_{m 0}=0.3$ as our lower bound (as suggested by large-scale galaxy flows [45]) we obtain $w_{0} \lesssim-0.6$ (or $\alpha \lesssim 4$ ) for the first initial data set and $w_{0} \lesssim-0.67$ (or $\alpha \lesssim 1.8$ ) for the second one. In both models, a larger lower bound on
$\Omega_{m 0}$, pushes the scalar field behavior toward that of a conventional cosmological constant.

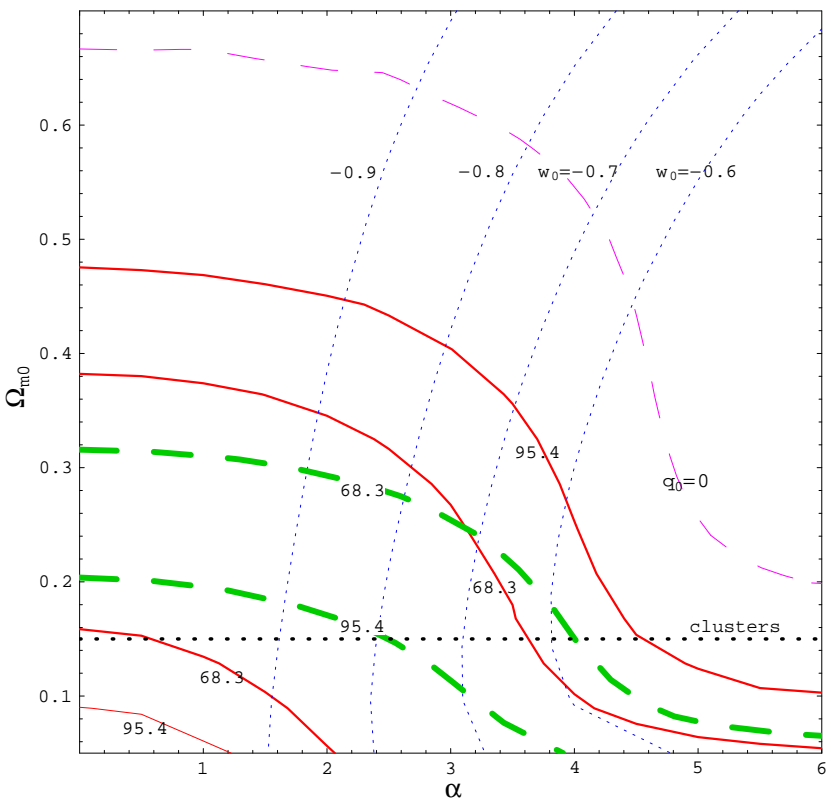

Figure 4. Contours of constant likelihood (95.4\% and $68 \%$ ) arising from lensing statistics (thick dashed contours) and type Ia supernovae are shown for the inverse power-law model. Also shown is the lower bound $\Omega_{m 0}=0.15$ from clusters and curves of constant present equation of state $w_{0}=p_{\phi 0} / \rho_{\phi 0}$. For the figure it is assumed that the field is frozen at the matter-radiation equality epoch

So far we have considered only flat models. For the sake of completeness we also obtained constraints on $\Omega_{m 0}$ and $\Omega_{\Lambda}$ in case we relax the flatness hypothesis and assume a conventional constant $\Lambda$. In Fig.6 we show the $95.4 \%$ and $68 \%$ C. L. limits from lensing (short dashed contours) and the SNeIa data on the parameters $\Omega_{m 0}$ and $\Omega_{\Lambda}$. In our lensing analysis we used $\Delta m=0.6 \mathrm{mag}$ for extinction. The lines $q_{0}=0$ (dashed) and $k=0$ (doted) are also displayed in Fig.6. The long dashed lines are roughly the $\pm 2 \sigma$ constraints from CMB anisotropy [6]. The pink shaded area is the best fitting region of the parameters. It is quite clear that current data favors an accelerated expansion (right of the $q_{0}=0$ line) and a nearly flat Universe. This region would be reduced even more if, for instance, we had considered other constraints on $\Omega_{m}$, as those coming from baryon fraction in clusters, clusters abundance and its evolution [7] or large-scale galaxy flows [45]. 


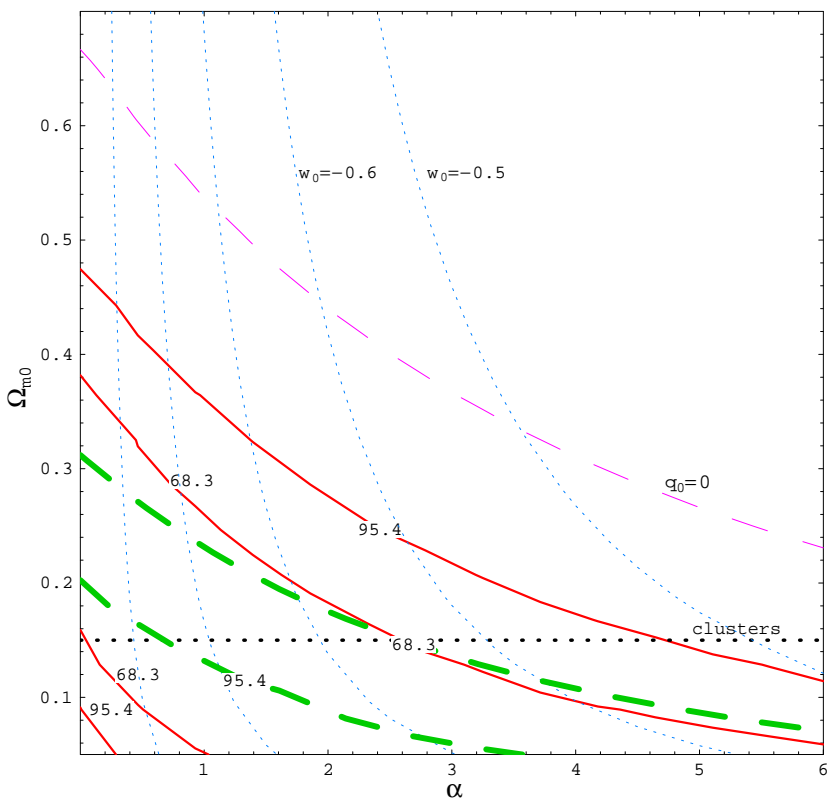

Figure 5. Contours of constant likelihood ( $95.4 \%$ and $68 \%$ ) arising from lensing statistics (thick dashed contours) and type Ia supernovae are shown for the inverse power-law model. Also shown is the lower bound $\Omega_{m 0}=0.15$ from clusters and curves of constant present equation of state $w_{0}=p_{\phi 0} / \rho_{\phi 0}$. For the figure it is assumed that the field starts tracking early in the Universe evolution.

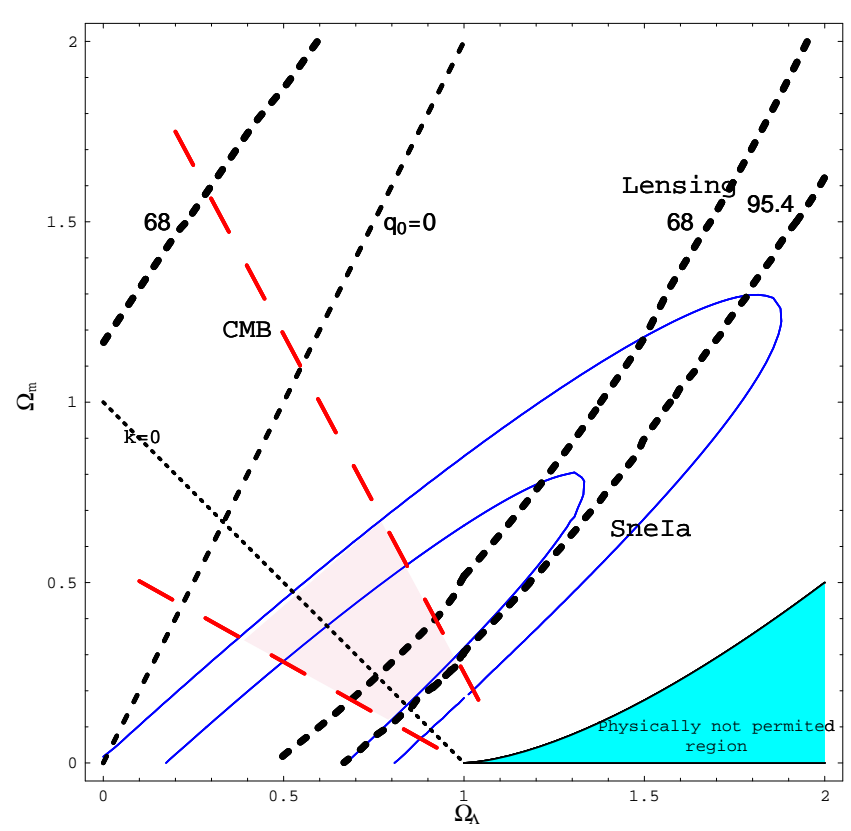

Figure 6. Contours of constant likelihood (95.4\% and 68\%) arising from lensing statistics (thick dashed contours) and type Ia supernovae are shown for open, flat and closed models with constant $\Lambda$. The lines $q_{0}=0$ (dashed) and $k=0$ (doted) are also displayed.

\section{Summary}

A consensus is beginning to emerge that we live in a nearly flat, low-matter-density Universe with $\Omega_{m 0} \sim$ 0.3 and a dark energy, negative-pressure component with $\Omega_{X} \sim 0.7$. The nature of this dark energy component is still not well understood; further developments will require deeper understanding of fundamental physics as well as improved observational tests to measure the equation of state at recent epochs, $w(t)$, and determine if it is distinguishable from that of the cosmological constant.

In this paper we considered observational constraints from lensing statistics and high-z SneIa on cosmological models whose matter content is nonrelativistic matter plus a negative-pressure dark energy component. We used a lensing approach, where extinction is considered, and that takes into account magnification bias and the selection function due to finite resolution and dynamic range in the configuration probability. For the SneIa analysis we considered data from the High-z Supernovae Search Team, the 27 low-z and 10 high-z SneIa reported in Ref. [1]. We used data with the MLCS method applied to the supernovae light curves. We showed that the two tests are compatible and observed that best fit models are in accelerated expansion $\left(q_{0}<0\right)$.

\section{Acknowledgments}

This is paper is based on work done with Josh Frieman and Ana Paula Miceli. I would like to deeply thank them. It is a pleasure to thank Luca Amendola and Franco Occhionero for several useful conversations and discussions during my visit to Rome last year. I would also like to thank Bharat Ratra and Silviu Podariu for sending their paper with SneIa constraints on inverse power-law models before publication and for kindly clarifying my understanding on several points. Many thanks to Cindy $\mathrm{Ng}$ for discussions and for her interest in our work. This work was supported by the Brazilian agencies $\mathrm{CNPq}$ and FAPERJ.

\section{References}

[1] A. G. Riess et al.,Astron. J. 116, 1009 (1998). P. M. Garnavich et al., Ap. J. 509, 74 (1998).

[2] S. Perlmutter et al., B.A.A.S., 29, 1351 (1997).

[3] S. Perlmutter et al., Ap. J. 517, 565 (1999).

[4] E. Harrison, Ap. J., 403, 28 (1993).

[5] S. Dodelson and L. Knox, astro-ph/9909454.

[6] A. Melchiorri et al., astro-ph/9911445. 
[7] N. A. Bahcall, J. P. Ostriker, S. Perlmutter and P. J. Steinhardt Science, 284, 1481 (1999). M. S. Turner, astro-ph/9901109.

[8] P.J.E. Peebles, Astrophys. J. 284, 439 (1984); M. S. Turner, G.Steigman, L. M. Krauss, Phys. Rev. Lett. 52, 2090 (1984).

[9] A. Einstein, Sitzungsberichte der Preussischen Akad. d. Wiss. 1917, 142 (1917).

[10] G. Lemaître, Mon. Not. R. astr. Soc. 90, 490 (1931).

[11] A. S. Eddington, Mon. Not. R. astr. Soc. 90, 668 (1930).

[12] V. Petrosian, E. Salpeter and P. Szekeres, Ap. J. 147, $1222(1967)$.

[13] J. R. Gunn, M. B. Tinsley, Nature 257, 454 (1975).

[14] J. D. North, The Measure of the Universe, Oxford: Clarendon Press (1965).

[15] B. Ratra and A. Quillen, Mon. Not. R. Astron. Soc., 259, 738, (1992); A. R. Cooray, A\&A, 342, 353 (1999).

[16] L. F. Bloomfield Torres and I. Waga, Mon. Not. R. Astron. Soc. 279, 712 (1996)

[17] V. Silveira and I. Waga, Phys. Rev. D 50, 4890 (1994); V. Silveira and I. Waga, Phys. Rev. D56, 4625 (1997)

[18] I. Waga and A. P. M. R. Miceli, Phys. Rev. D, 59, 103507 (1999).

[19] Y. B. Zeldovich, Sov. Phys. Uspekhi, 11 , 381 (1968).

[20] W.H. McCrea, Proc. Roy. Soc. London A 206, 562 (1951).: V. Petrosian, in Confrontation of Cosmological Theories with Observational Data, ed. M. S. Longair (Dordrecht: Reidel), 31 (1974).; B. M. Tinsley, Physics Today, June, 32 (1977).; J. E. Felten and R. Isaacman, Rev. Mod. Phys. 58, 689 (1986).;P. J. E. Peebles, Publ. Astron. Soc. Pac. 100, 670 (1988).; S. Weinberg, Rev. Mod. Phys. 61, 1 (1989); L. Abbot, Sci. Am. 258(5), 427 (1989).; S. M. Carrolll, W. H. Press and E. L. Turner, Annu. Rev. Astron. Astrophys., 30, 499 (1992).; For a recent review see V. Sahni and A. Starobinsky, astro-ph/9904398.

[21] J. Frieman, C. Hill, and R. Watkins, Phys. Rev. D 46 , $1226(1992)$.

[22] M. Fukugita and T. Yanagida, preprint YITP/K-1098 (1995)

[23] J. A. Frieman, C. T. Hill, A. Stebbins and I. Waga, Phys. Rev. Lett. 75, 2077 (1995).

[24] K. Coble, S. Dodelson, and J. A. Frieman, Phys. Rev. D 55, 1851 (1997).

[25] J. A. Frieman and I. Waga, Phys. Rev. D 57, 4642 (1998)

[26] R. R. Caldwell, R. Dave, and P.J. Steinhardt, Phys. Rev. Lett. 80, 1582 (1998).

[27] B. Ratra and P. J. E. Peebles, Phys. Rev. D 37, 3407 (1988); P. J. E. Peebles and B. Ratra, Ap.J. 325, L17 (1988)

[28] M. Ozer and M. O. Taha, Nucl. Phys. B 287, 776 (1987); K. Freese et al., Nucl. Phys. B 287, 797 (1987); M. Reuter and C. Wetterich, Phys. Lett. B188, 38
(1987): W. Chen and Y. S. Wu, Phys. Rev. D41, 695 (1990); J. C. Carvalho, J. A. S. Lima and I. Waga, Phys. Rev. D46, 2404, (1992); I. Waga, Ap. J. 414, 436; (1993); J. M. Overduin and F. I. Cooperstock, Phys. Rev. D58, 043506, (1998).

[29] A. Vilenkin, Phys. Rev. Lett., 53, 1016 (1984); J. N. Fry, Phys. Lett. B158, 211 (1985); H. A. Feldman and A. E. Evrard, Int. J. Mod. Phys. D 2, 113 (1993); J. Stelmach and M. P. Dabrowski, Nucl. Phys. B 406, 471 (1993); H. Martel, Ap. J. 445, 537 (1995); L. M. A. Bittencourt, P. Laguna, R. A. Matzner, hep-ph/9612350; M. Kamionkowski and N. Toumbas, Phys. Rev. Lett., 77, 587 (1997); D. N. Spergel and U. L. Pen, Ap. J. 491, L67 (1997); M. S. Turner and M. White, Phys. Rev. D56, R4439 (1997):W. Hu, Ap. J. 506, 495 (1998); S. Perlmutter, M.S. Turner and M. White, Phys. Rev. Lett. 83, 670 (1999); M. Bucher and D. Spergel Phys. Rev. D60, 043505, (1999): L. Wang, R. R. Caldwell, J. P. Ostriker and P. J. Steinhardt, astro-ph/9901388.

[30] M. White, Ap. J. 506, 485 (1998).

[31] C. Wetterich, Nuclear Physics B, 302, 668 (1988); V. Sahni, H. A. Feldman and A. Stebbins, Ap. J. 385, 1 (1992); P. T. P. Viana and A. R. Liddle, Phys. Rev. D 57, 674 (1998); P. Ferreira and M. Joyce, Phys. Rev. Lett., 79, 4740 (1997): Phys. Rev. D 58, 023503 (1998): A. Liddle and R. Scherrer, Phys. Rev. D 59, 023509 (1999): J. Uzan, Phys. Rev. D 59, 123510 (1999); P. Binétruy, Phys. Rev D 60, 063502 (1999): A. Masiero, M. Pietroni and F. Rosati, hep-ph/9905346; L. Amendola, astro-ph/9908023; astro-ph/9908023: A. Albrecht and C. Skordis, astro-ph/9908085; V. Sahni and L. Wang, astro-ph/9910097;

[32] I. Zlatev, L. Wang and P. J. Steinhardt, Phys. Rev. Lett., 82, 896 (1999); P. J. Steinhardt, L. Wang and I. Zlatev, Phys. Rev D 59, 123504 (1999).

[33] S. M. Carroll, Phys. Rev. Lett, 81, 3067 (1998); C. Kolda and D. H. Lyth, Phys. Rev. Lett B 458, 197 (1999); K. Choi, hep-ph/9912218.

[34] S. Podariu and B. Ratra, astro-ph/9910527.

[35] D. Maoz et al., Ap. J. 409, 28 (1993); D. Crampton, R. D. McClure, and J. M. Fletcher, Ap. J. 392, 23 (1992); H. K. C. Yee, A. V. Filipenko, and D. H. Tang, A. J. 105, 7 (1993); J. Surdej et al. ibid. 105, 2064 (1993); E. E. Falco, in Gravitational Lenses in the Universe, edited by J. Surdej, D. Fraipont-Caro, E. Gosset, S. Refsdal, and M. Remy (Liege: Univ. Liege), 127 (1994); C. S. Kochanek, E. E. Falco, and R. Shild, Ap. J. 452, 109 (1995); A. O. Jaunsen et al., A\&A 300, 323 (1995).

[36] E. L. Turner, J. P. Ostriker, J. R. Gott III, Ap. J. 284. 1 (1984).

[37] S. M. Faber and R. E. Jackson, Ap. J. 204, 668 (1976).

[38] E. L. Turner, Ap. J. 365, L43 (1990); M. Fukugita and E. L. Turner, Monthly Notices Roy. Astron. Soc. 253, 99 (1991).

[39] C. S. Kochanek, Ap. J. 419, 12 (1993).

[40] C. S. Kochanek, Ap. J. 466, 47 (1996).

[41] E. E. Falco, C. S. Kochanek and J. A. Muñoz, Ap. J. 494, 47 (1998). 
[42] R. O. Marzke, M. J. Geller, J. P. Huchra and H. G. Corvin, AJ. 108, 437 (1994).

[43] S. Perlmutter et al., Ap. J. 483, 565 (1997).

[44] A. G. Riess, W. H. Press and R. P. Kirchner, Ap. J. 473, 88 (1996).
[45] I. Zehavi and A. Dekel, Nature 401, 252 (1999).

[46] I. Waga and J. A. Frieman, submitted to Phys. Rev. D $(2000)$. 